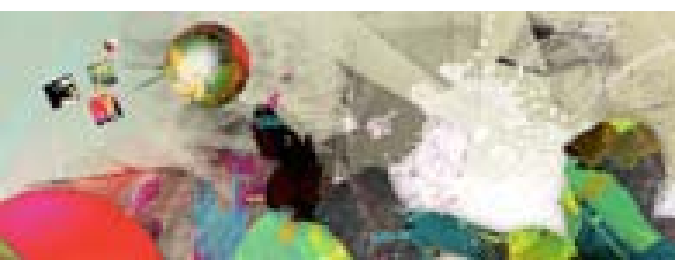

\title{
Representaciones de la sociedad venezolana en los discursos del Presidente de Venezuela José Tadeo Monagas
}

\section{Representations of the Venezuelan society in the discourse of President José Tadeo Monagas}

\author{
Carmen Valecillos Vázquez ${ }^{(*)}$ \\ Universidad de Los Andes-Táchira - Venezuela \\ cardeval@gmail.com \\ Francisco Bernete ${ }^{(*)}$ \\ Universidad Complutense de Madrid - España \\ fbernete@ccinf.ucm.es
}

\begin{abstract}
Resumen
El presente artículo identifica las representaciones que José Tadeo Monagas, presidente de Venezuela en el siglo XIX, ofrece sobre la sociedad venezolana en sus discursos anuales ante el Parlamento. Los relatos, en este análisis, se estudian como productos enculturizadores que proporcionan modelos axiológicos y cognitivos con el objetivo de mediar entre lo que sucede y la interpretación que las personas hacen sobre la realidad. Las categorías usadas se obtienen a partir de un análisis de contenido con el cual se registran los referentes
\end{abstract}

\begin{abstract}
The present article identifies the representations that Jose Tadeo Monagas, president of Venezuela in the nineteenth century, offers about the Venezuelan society in his annual discourses before Parliament. In this analysis, the narrations are understood as enculturation products, which provide axiological and cognitive models with the objective of mediating between what is happening and the interpretation that people make about reality. The categories used are obtained from a content analysis, which
\end{abstract}


y su representación en los ocho discursos estudiados. Los resultados reflejan que las representaciones con que José Tadeo Monagas narra la realidad venezolana se encuentran usualmente disociadas de los hechos y acontecimientos ocurridos en el período estudiado, con el objetivo de evitar las explicaciones sobre los cambios que se producen y los problemas que la sociedad venezolana experimenta en ese momento histórico.

Palabras clave: representaciones, discurso presidencial, José Tadeo Monagas, sociedad venezolana, mediación, análisis de contenido. has been employed to register the referents and their representation in the eight speeches studied. The results show that José Tadeo Monagas' representations of Venezuela's reality are usually divorced from the facts and events of the period studied, in order to avoid explaining the changes that occur and the problems of Venezuelan society in that historical moment.

Keywords: representations, presidential discourse, José Tadeo Monagas, Venezuelan society, mediation, content analysis. 
Este trabajo se ha realizado al mismo tiempo que una investigación doctoral, donde se analizan los discursos de los 10 últimos Jefes de Estado de Venezuela, cuando se dirigen al Congreso de la República en sus discursos anuales de Memoria y Cuenta. El presidente José Tadeo Monagas (en adelante, JTM) gobernó en el siglo XIX. Por tanto, el análisis de sus discursos es colateral e inédito, realizado expresamente para esta publicación ${ }^{1}$.

JTM fue electo como Presidente de Venezuela en dos ocasiones: en la primera para gobernar de 1847 a 1851 y en la segunda para ejercer ese mismo cargo de 1855 a 1859 , período que no culminó pues presentó su renuncia en 1858 cuando la Revolución de Marzo, comandada por Julián Castro, tomó Caracas. Por ello, convertimos en objeto material de análisis los ocho discursos que pronunció efectivamente como Presidente: 1848, $1849,1850,1851,1855,1856,1857$ y $1858^{2}$.

En esos discursos ante el Congreso, el Presidente transmite una visión de la realidad venezolana que se compadece mal con la situación del país durante sus periodos de gobierno. Y, sin embargo, no aparece una contradicción manifiesta, puesto que el emisor de los discursos no sitúa en el mismo plano los acontecimientos (entre ellos, los violentos) que sacuden a la sociedad venezolana y la representación de los referentes institucionales de su Memoria y Cuenta (usualmente benigna, si se exceptúa la de los adversarios). Nuestra hipótesis de partida es que el sujeto de la enunciación ha entendido que era la manera en la cual podía hacer un discurso mediador que le resultara funcional

Para Manuel Martín Serrano el uso funcional de los discursos mediadores puede trazarse tres objetivos: 1. Mejorar su eficiencia, 2. Disminuir o eliminar las disfunciones, y 3. Evitar o posponer el ajuste histórico necesario (Martín Serrano, 2004: 76). En los discursos del Presidente Tadeo veremos si se trazan tales objetivos.

1 Los discursos utilizados se extrajeron del Repertorio Histórico Biográfico del General José Tadeo Monagas (1983).

2 El Congreso Nacional de la República de Venezuela recibió en el año 1955 dos mensajes presidenciales, el primero de José Gregorio Monagas quien entregó en el mes de enero la Presidencia y el otro de José Tadeo Monagas quien presentó su mensaje el 14 de febrero, después de haberse juramentado el 31 de enero como mandatario para el período 1855-1859. Por tanto, el discurso de este primer año de gobierno es el único que no cumple con el requisito de ser mensaje de Memoria y cuenta. 


\section{CONTEXTUALiZación HistóRica}

JTM es un buen ejemplo de la figura caudillista que gobernó en varios países latinoamericanos en la segunda mitad del siglo XIX y un representante de esos caudillos que ostentaron el poder político o militar en Venezuela luego de la guerra de la Independencia y con el prestigio de haber sido parte del ejército de Simón Bolívar. Nació de una familia económicamente estable, dueña de haciendas en el oriente del país. Militarmente se formó en el ejército patriota, primero, bajo el mando del general Santiago Mariño, posteriormente bajo las órdenes del mismo Simón Bolívar: "Su habilidad como jinete, su destreza con la lanza (...) su inteligencia metódica y su valor innato, le van a permitir liderar las feroces montoneras que lo acompañarán en la lucha por la independencia...” (Alarico, 2006: 12).

Compartió y defendió las ideas bolivarianas en casi toda su vida pública; sin embargo, también vaciló al momento de grandes decisiones, como en la separación de Venezuela de la Gran Colombia para el año 1830. Tuvo una vida de combatiente, se inició en la guerra en 1813 y murió unos meses después que triunfara en junio de 1868 en la de la Revolución Azul (Alarico, 2006: 108; Rodríguez, 2002: 224).

La mayor parte de su vida la pasó en plena lucha: primero por la independencia de Venezuela, luego por la consolidación de la República, más tarde defendiendo su gobierno y, finalmente, por alcanzar el poder nuevamente. Entre 1784 y 1868, años del nacimiento y la muerte del caudillo, Venezuela se encontraba sumida en la guerra, con los sistemas productivos devastados, un país carente por completo de instrucción pública primaria o de un sistema de salubridad que paliara la situación del campesino y las clases menos favorecidas, carente de todo orden en la administración pública y de un sistema de gobierno que pudiera enfrentar los males más terribles de la población: latifundismo, endemias, analfabetismo y esclavitud o cuasi esclavitud de la mayoría de los campesinos que tenían miserables condiciones de vida. Esa fue la realidad en la que gobernó JTM, una realidad enfrentada a diario por 1.273 .155 venezolanos, habitantes de un país con ingresos algo superiores a 2 millones de pesos y con una deuda pública mayor a 20 millones de pesos (Alarico, 2006: 57).

JTM llega a ser presidente de Venezuela porque recibe el respaldo del líder conservador y ex-presidente de Venezuela, José Antonio Páez, pero

Mediaciones Sociales, № 10, I semestre 2012, pp. 63-85. ISSN electrónico: 1989-0494.

DOI: http://dx.doi.org/10.5209/rev_MESO.2012.n10.39682 
también por cuatro características presentes en su trayectoria militar y política: prestigio político, liderazgo militar, supervivencia política y éxito en sus proyectos de oriente, es decir, en su territorio (Mangano, 2010: 56).

El período en que gobiernan JTM y su hermano José Gregorio se conoce en la historia venezolana como el Monagato. Los hermanos Monagas gobiernan durante 11 años a partir de 1847, con claros visos de nepotismo tanto en la presidencia, cuando un hermano reemplaza al otro, como en la administración pública central y local, donde nombran a familiares y amigos para el desempeño de los cargos más importantes.

A continuación se mencionan algunas características distintivas del gobierno de José Tadeo en sus dos períodos:

a. JTM cuenta inicialmente con el respaldo del partido conservador (liderado por José Antonio Páez), luego se separa de éste y establece un proyecto liberal.

b. La separación definitiva de la tendencia liberal se produce después de un hecho sangriento: "el asesinato del Congreso" o "el fusilazo", cuando en un tumulto los liberales toman el Congreso dominado hasta ese momento por la fuerza conservadora. Allí mueren varios líderes conservadores y pierde su autonomía el Poder Legislativo.

c. Comienza su gobierno en una Venezuela sometida a una grave crisis agropecuaria que se mantiene casi permanentemente durante todo su mandato.

d. Se caracteriza por ser un período extraordinariamente violento, que vive una guerra civil no declarada, expresada en varios alzamientos o rebeliones ${ }^{3}$.

e. Se intenta por primera vez consolidar una fuerza militar del Estado.

f. Se declara continuamente el respeto a las leyes y la intención de mejorar la administración pública y judicial.

3 En 1848, cuando José Antonio Páez enfrenta en los llanos a José Cornelio Muñoz, enviado por Monagas a defender el gobierno; en 1849 en Coro, levantamiento sometido por el general José Laurencio Gómez, (en el gobierno de José Gregorio se producen rebeliones en los años 1853 y 1854), y en 1858 con el levantamiento de Julián Castro, gobernador de Valencia, quien lideró la Revolución de Marzo y asumió el poder en ese mismo mes. 
La mejor descripción de la situación vivida por la población venezolana durante el Monagato la ofrece el obispo venezolano Mariano de Talavera (1777-1861) quien en la toma de posesión del segundo gobierno de JTM le resume a éste la situación en que se encuentra el país:

"Señor, los males físicos, morales y políticos se han confederado para oprimir esta desgraciada República: Carestía de las subsistencias... lamentable atraso de la agricultura... reclamaciones casi amenazadoras por parte de algunas potencias extranjeras... enfermedades y epidemias... silencio sepulcral de la prensa... erario exhausto... una deuda inmensa... el agio llevado hasta el escándalo... la justicia envilecida... las garantías violadas... robos sacrílegos y asesinatos nocturnos... disensiones civiles, opiniones encontradas, odios recíprocos... la guerra entre hermanos" (citado en Alarico, 2006: 79).

\section{EL DISCURSO VISTO COMO ACTIVIDAD MEDIADORA}

En esta investigación los discursos se estudian como elementos mediadores en el contexto socio-político en que se producen. El paradigma de la mediación desarrollado por el profesor Manuel Martín Serrano plantea que los mensajes proporcionan una visión de los hechos, pasados o presentes, y esa visión se puede descifrar a partir de los datos de referencia con los que se describe el acontecer. El concepto expresado por Martín Serrano (2008: 76) es el siguiente: "La mediación se define como la actividad que impone límites a lo que puede ser dicho, y a las maneras de decirlo, por medio de un sistema de orden”. Y Miquel Moragas i Spá, siguiendo a Martín Serrano, dirá de la mediación social:

"Desde el punto de vista cognitivo, la mediación equivaldría al sistema de reglas y de operaciones aplicadas a cualquier conjunto de hechos, o de cosas pertenecientes a planos heterogéneos de la realidad, para introducir un orden. La función mediadora permite analizar las formas de control. La función mediadora (de control) se concreta como función transmisora de comunicación” (Moragas i Spá, 2011: 43).

Por tanto, los relatos se analizan como productos enculturizadores que proporcionan modelos axiológicos y cognitivos con el objetivo de mediar entre lo que sucede y la interpretación que las personas hacen sobre la realidad (Martín Serrano, 2004: 48). En este caso, son elementos mediadores que proceden de la Presidencia de la República, como otros proceden de otras

Mediaciones Sociales, № 10, I semestre 2012, pp. 63-85. ISSN electrónico: 1989-0494. DOI: http://dx.doi.org/10.5209/rev_MESO.2012.n10.39682 
instituciones que ejercen funciones mediadoras: familia, iglesias, partidos, medios de comunicación o escuelas. Así, los discursos políticos, como parte de los mensajes públicos que se emiten desde una institución, también tienen como finalidad mediar entre la realidad y la percepción que los destinatarios construyen sobre ella. Para cumplir esa función mediadora, seleccionan datos (relativos a los lugares, acontecimientos, personajes, etc.) y una organización de los datos en la narración. "La mediación propone representaciones del tiempo, del espacio y de lo que acontece. Logra que nuestra conciencia se historice, es decir, que encuadre el conocimiento de la realidad en modelos históricamente determinados" (Martín Serrano, 2004: 56).

Las representaciones, como conjunto de datos de referencia, se plasman en relatos elaborados en un tiempo, un espacio y un determinado contexto de acontecimientos con el objetivo de que sean interiorizadas como representaciones sociales que orienten la acción social, según el autor: "El Mediador [en este caso el Jefe de Estado] se enfrenta con la tarea de sugerir una interpretación socialmente aceptable de lo que acontece entre el repertorio de las representaciones del mundo que posee el grupo" (Martín Serrano, 2004: 216). Para construir esa interpretación el Mediador usa datos y hecho reales que ilustren y/o demuestren que su visión de la realidad (representación) sigue siendo válida para el contexto que él señala. Por ello, Martín Serrano es concluyente al sentenciar:

"La producción social de comunicación aparece, a la luz de estas reflexiones, como una actividad destinada a reproducir y producir una clase de representaciones sociales adecuadas para enfrentarse con el cambio de las condiciones objetivas que afronta la comunidad, manteniendo la validez de los principios más universales en los que se apoya la vida en común” (Martín Serrano, 2004: 217).

\section{EL DISEÑO DE LA INVESTIGACIÓN}

Para identificar las representaciones (los datos de referencia y su organización) se diseñó un análisis de contenido ${ }^{4}$, fragmentando el corpus

\footnotetext{
4 Este estudio asume las directrices que da Laurence Bardin cuando define el análisis de contenido como: "Un conjunto de técnicas de análisis de comunicaciones tendente a obtener indicadores (cuantitativo o no) por procedimientos sistemáticos y objetivos de descripción del contenido de los mensajes permitiendo la inferencia de conocimientos relativos a las condiciones de producción/recepción (variables inferidas) de estos mensajes” (Bardin, 2002: 32).
} 
(los 8 discursos) en unidades de análisis. Se tomó como unidad de análisis las descripciones de alguno de los referentes contemplados (individuos, grupos o instituciones), siempre que a propósito de ese referente en el discurso se aportase alguno de los datos contemplados como "categorías". Posteriormente, se agruparon las posibles categorías o datos atribuidos al referente en cuatro bloques:

- cualidades (físicas, de valía personal, de sociabilidad, políticas y culturales),

- deberes,

- relaciones con otros actores (de oposición, de alianza, de sumisión o de dominio)

- y comportamientos.

El repertorio de los referentes (y también de las categorías recogidas) se elaboró con base en la lectura previa de todos los discursos presidenciales, pues ello permitía identificar los que, con cierta frecuencia, aparecían en los relatos. En este caso, inicialmente se encontraron nueve referentes sobre los que frecuentemente José Tadeo Monagas emite valoraciones o descripciones. El análisis, para el desarrollo de este artículo, se ha centrado en seis de ellos: CONGRESO, PRESIDENTE, PAÍS, PUEBLO, GOBIERNO y ADVERSARIOS.

Todo el proceso de categorización mencionado anteriormente se inicia con la identificación en los discursos de expresiones literales con las que se atribuyen rasgos a los diferentes referentes y estas expresiones se convierten en categorías (véase Tabla 1).

Tabla 1. Ejemplos de categorización a partir de las expresiones literales

\begin{tabular}{|l|l|}
\hline \multicolumn{1}{|c|}{ Expresiones literales del texto } & Categorías para el análisis \\
\hline Egoísta, vela solo por sus intereses & Egoísta \\
\hline Criminal, ladrón, comete actos injustos & Criminal \\
\hline Faccioso, busca la división & Faccioso \\
\hline Bondadoso, amable, cariñoso, entregado & Bondadoso \\
\hline Empobrecido, atrasado, débil, en la miseria & Atrasado \\
\hline
\end{tabular}

Mediaciones Sociales, № 10, I semestre 2012, pp. 63-85. ISSN electrónico: 1989-0494. DOI: http://dx.doi.org/10.5209/rev_MESO.2012.n10.39682 


\section{RESULTADOS DEL ANÁLISIS DE CONTENIDO}

Presentamos, en primer lugar, los rasgos cualitativamente diferentes que se encuentran atribuidos a los nueve referentes de los que con mayor frecuencia se ofrecen datos en los discursos.

\section{Tabla 2. Referentes y rasgos atribuidos en los discursos de José Tadeo Monagas}

\begin{tabular}{|l|l|}
\hline \multicolumn{1}{|c|}{ Referentes } & $\begin{array}{l}\text { Rasgos distintos que se le atribuyen y (entre paréntesis) frecuencia de } \\
\text { aparición de los rasgos }\end{array}$ \\
\hline ESTADO & Atrasado (3) \\
\hline BOLÍVAR & $\begin{array}{l}\text { Visionario (8), Genio (5), Libertador (3), Soldado (1), Amigo del Presidente } \\
(1)\end{array}$ \\
\hline ALIADOS & $\begin{array}{l}\text { Valiente (7), Virtuoso (4), Se somete a Gobierno (2), Defiende leyes (2), Se } \\
\text { somete a Pueblo (1), Defiende la Constitución (1) }\end{array}$ \\
\hline ADVERSARIOS & $\begin{array}{l}\text { Manipulan la información (12), Se oponen a País (12), Criminales (9), Egoís- } \\
\text { tas (8), Se oponen a Gobierno (6), Se oponen a Pueblo (6), Facciosos (3) }\end{array}$ \\
\hline GOBIERNO & $\begin{array}{l}\text { Cumple y hace cumplir las leyes (8), Se adhiere a Pueblo (8), Democrático } \\
(6), \text { Dialogante (5), Generoso (4), Somete al enemigo (4), Justo (3), Respeta } \\
\text { las instituciones (3), Enfrenta dificultades (3), Honesto (2), Firme (2) }\end{array}$ \\
\hline CONGRESO & $\begin{array}{l}\text { Deben legislar (32), Deben buscar el progreso y la paz (20), Honorables } \\
\text { (13), Ilustrados (13), Patriotas (9), Se adhieren a Pueblo (4), Eficaces (3), } \\
\text { Bondadosos (3), Garantizan justicia y libertad (2), Creyentes (1), Falibles } \\
(1), \text { Se adhieren a Presidente (1) }\end{array}$ \\
\hline PAÍS & $\begin{array}{l}\text { Estable (16), Busca orden, progreso y paz (9), Amigo de otros países (7), } \\
\text { Enfrenta dificultades (7), Democrático (6), Grande (6), Se adhieren a Go- } \\
\text { bierno (6), Joven (3), Depende de Congreso (3), Atrasado (3), Independiente } \\
(2), \text { Leal (2), Depende de pueblo (1), Bondadoso (1), }\end{array}$ \\
\hline PUEBLO & $\begin{array}{l}\text { Bondadoso (20), Democrático (11), Ilustrado (11), Se adhiere a gobierno } \\
\text { (11), Merece y busca mejoras (9), Víctima (8), Patriota (7), Optimista (5), } \\
\text { Creyente (5), Valiente (4), Soberano (4), Se adhiere a Presidente (4), Extra- } \\
\text { viado (4), Depende de Congreso (3), Glorioso (3), Se opone a Adversarios } \\
(2), \text { Depende de Presidente (1) }\end{array}$ \\
\hline PRESIDENTE & $\begin{array}{l}\text { Creyente (15), Patriota (11), Cumple la ley (11), Busca orden, progreso y } \\
\text { paz (9), Se adhiere a pueblo (8), Bondadoso (7), Firme (6), Ilustrado (6), } \\
\text { Responsable (6), Colaborador (5), Servidor (5), Democrático (5), Defiende la } \\
\text { propiedad privada (4), Honesto (4), Leal (4), Autocrítico (3), Enfrenta difi- } \\
\text { cultades (3), Valiente (2) }\end{array}$ \\
\hline
\end{tabular}

Mediaciones Sociales, № 10, I semestre 2012, pp. 63-85. ISSN electrónico: 1989-0494. DOI: http://dx.doi.org/10.5209/rev_MESO.2012.n10.39682 
José Tadeo Monagas en sus discursos de Memoria y Cuenta alude a los nueve referentes indicados. De ellos, son seis los descritos con más abundancia de rasgos y, a su vez, tres de ellos destacan por la cantidad de rasgos distintos que le son atribuidos: "PRESIDENTE", "PUEBLO" y “CONGRESO”.

\subsection{Consideraciones generales sobre las atribuciones más frecuentes}

1) La abundancia de los rasgos atribuidos tanto a PRESIDENTE como a PUEBLO, puede interpretarse como indicador del personalismo con que se gobernaba al país durante el siglo XIX, tras la independencia, y que se prolongaría en las primeras décadas del siglo XX. El fenómeno, que no es objeto del presente estudio, se ha definido como caudillismo, sobre el cual Sabino señala:

"Carente de una tradición de gobierno propio durante la Colonia, y devastada por luchas intestinas que a veces adquirieron singular pugnacidad, Venezuela recorrió reiteradamente durante el siglo pasado un ciclo en el que se alternaban situaciones de anarquía, caracterizadas por gobiernos débiles y de escasa representatividad, con dictaduras más o menos consolidadas, que derivaban rápidamente en un sistema autoritario y por completo personalista. Caudillismo, lucha civil y anarquía fueron así las notas predominantes de un largo período que concluyó, aunque no completamente, con el fin de la dictadura de Juan V. Gómez, ocurrida cuando éste murió a finales de 1935” (Sabino, 1995: 1).

2) El papel dado al CONGRESO en los discursos de JTM también puede encontrar una explicación en el proceso político que vivía Venezuela para la época. Sabino lo expresa con estas palabras: "Las constituciones elaboradas durante ese siglo [XIX] reconocieron, por lo general, la estructura caudillista del poder: establecieron por lo general un ejecutivo fuerte y una representación de tipo indirecto, basada sobre elecciones de segundo grado, para los cuerpos legislativos" (1995: 1).

La representación del CONGRESO focalizada en una amplia tarea para legislar y buscar el progreso entra en contradicción con los acontecimientos políticos del momento y con la nula autonomía que a partir de 1848 tuvo el Poder Legislativo durante el Monagato. Históricamente en Venezuela se lamentan los hechos ocurridos el 24 de enero de 1848 cuando mueren varios parlamentarios, en la sede del Congreso Nacional, como consecuencia

Mediaciones Sociales, № 10, I semestre 2012, pp. 63-85. ISSN electrónico: 1989-0494.

DOI: http://dx.doi.org/10.5209/rev_MESO.2012.n10.39682 
de una manifestación violenta generada por un grupo de liberales. Al respecto señala Arráiz:

"Monagas, alertado acerca de los hechos en curso, se presenta a caballo, acompañado del general Santiago Mariño, y con las fuerzas del orden restablece la calma. La herida para el Poder Legislativo había sido mortal. El parlamento después de estos hechos tardó años en recuperar autonomía. Monagas cada vez más lo doblegó como un apéndice de su propio mando" (Arráiz, 2008: 47).

2.1) En el caso del deber de legislar, asignado únicamente a CONGRE$\mathrm{SO}$, el deber va un poco más allá de elaboración o modificación de leyes pues implícitamente involucra la obligación de otras acciones. Por ejemplo, José Tadeo Monagas en el discurso de 1849 pide a los congresistas elaborar un cuerpo de leyes "que deroguen todos los actos de la pasada tiranía, que purifiquen la Administración política, que organicen la justicia, que metodicen las rentas, que restauren y consoliden el crédito público, que hagan justicia a todos los derechos y definan nuestros deberes”. Ese deber lo debe cumplir un cuerpo legislativo que ha sido el resultado del asalto y la disolución del Congreso originario.

2.2) También el deber de legislar está muy relacionado con el otro deber que siempre se le adjudica al CONGRESO: Velar por el progreso, el orden y la paz del país. No obstante, en este segundo deber sí involucra JTM a otros actores de la sociedad venezolana: Responsables del progreso, orden y paz también son, con frecuencia, el PAÍS y el PRESIDENTE. Estos actores esperan ese estado ideal o lo buscan, sin embargo, de esta responsabilidad queda excluido el GOBIERNO, al que nunca se le atribuye esa responsabilidad.

3) El único atributo que siempre está asignado al PAÍS, y a ningún otro referente, es el rasgo de Estable. Es importante esta asociación por la contradicción que se genera con la realidad venezolana. José Tadeo Monagas gobernó sofocando rebeliones, enfrentando las consecuencias de ellas y paliando las situaciones de pobreza e inestabilidad que generaban. En el año 1849 decía: "La Nación marcha en paz y bajo los auspicios de ésta llegará al punto que tiene señalado en EL GRAN LIBRO DEL DESTINO (sic.)" e igualmente, en el año 1857 pocos meses antes de renunciar (a causa del triunfo que alcanzaba la Revolución de Marzo iniciada por Julián

Mediaciones Sociales, № 10, I semestre 2012, pp. 63-85. ISSN electrónico: 1989-0494. DOI: http://dx.doi.org/10.5209/rev_MESO.2012.n10.39682 
Castro en Carabobo) repetía: "Yo me congratulo con la República entera, y la felicito, por haber llegado en esta época venturosa, que el hecho mismo de vuestra reunión a la sombra de la paz que disfrutamos es el gaje cierto y solemne de que nuestras esperanzas no serán vanas".

4) Otro atributo destacado es la cualidad de Bondadoso que se le asigna frecuentemente a cuatro de los nueve referentes: CONGRESO, PAÍS, PRESIDENTE y, especialmente, PUEBLO (en veinte ocasiones y en seis de los ocho discursos). Así mismo, el rasgo de Democrático también se atribuye a cuatro referentes: GOBIERNO, PAÍS, PRESIDENTE y PUEBLO. En esta ocasión la frecuencia del rasgo es menor.

5) Patriota, Ilustrado y Creyente son rasgos atribuidos frecuentemente a CONGRESO, PRESIDENTE y PUEBLO. Sin embargo, la Honorabilidad es una cualidad asignada únicamente al CONGRESO.

6) Del campo de comportamientos podemos decir que los negativos se atribuyen en exclusiva al referente ADVERSARIOS (categoría de análisis que encierra a los revoltosos, a los rebeldes, a líderes opositores, a partido liberal). También con frecuencia se asigna a estos actores únicamente las características de Criminales, Egoístas y Facciosos. En 1849 dice JTD que sus adversarios se mantienen "en el empeño criminal y deshonroso de engañar al mundo, de combatir a su patria" y en el discurso de 1858 sigue señalando que para desprestigiar al gobierno dentro y fuera del país: "se recurría, en fin, a cuantas artes pudieran inventar, en siniestro maridaje, la malicia y la calumnia, para mantener la sociedad en constante inquietud". Como se puede observar la acción de Manipular información se conjuga con las características de Criminales, Egoístas y Facciosos que se le da a todo aquel que haga oposición a su gobierno.

7) José Tadeo Mongas en sus ocho discursos maneja relaciones de oposición o enfrentamiento, de alianza o adhesión, sometimiento o subordinación, así como de dominio de un actor sobre otro. No obstante, las que por su frecuencia destacan son las de Adhesión al gobierno y la de Oposición al País, la primera relación se establece entre el PUEBLO y el PAÍS, referentes estos que, según JTM, son los que establecen alianzas con el GOBIERNO, excluyendo al resto de actores. Mientras que los ADVERSARIOS son los únicos que Enfrentan al país y también se oponen al GOBIERNO y al PUEBLO. 
8) Aunado a que del campo de relaciones escoge la dependencia para definir al PUEBLO, junto a unas cualidades muy positivas (Bondadoso, Ilustrado, Democrático, Glorioso, Optimista, Patriota, Valiente y Soberano) también agrega las de Victima y Extraviado: "Pero no le han redimido de grandes sufrimientos y ha sido frecuentemente la víctima del despotismo hipócrita o de la artificiosa demagogia" y "Los venezolanos desgraciadamente extraviados con el nombre de oligarcas encontraron cabida para continuar trillando su senda de conspiración", dice José Tadeo Monagas. Se suma a esta representación la dependencia del CONGRESO y del PRESIDENTE. Señala JTM en 1848: "Los pueblos ansían por recibir aquellas medidas saludables que su situación exige y sólo pueden derivar de la fuente abundante y pura del Poder Legislativo" y en 1855 en la frase introductoria de su discurso señala que el PUEBLO pone en manos del CONGRESO todas sus esperanzas.

\subsection{Las representaciones de los distintos referentes}

El análisis de los rasgos atribuidos con mayor frecuencia a cada referente en los ocho discursos analizados, proporciona resultados reveladores de la visión que expresa el Presidente de la República sobre cada uno de los referentes que siguen:

\subsubsection{La representación del CONGRESO y/o LOS CONGRESISTAS}

El atributo de mayor presencia registrada (en 32 ocasiones) es el deber que tienen los legisladores de elaborar instrumentos legales que ordenen la administración pública y el país en general. Sumado a esto, el Primer Mandatario manifiesta la necesidad de que los legisladores a través de ese ordenamiento jurídico lleven el progreso y la paz a la nación (en 20 ocasiones). Es el CONGRESO, el único referente al que se le asignan obligaciones; así quedan exentos de ellas GOBIERNO y PRESIDENTE, quienes por la naturaleza de sus responsabilidades deberían ser corresponsables en estos y otros compromisos. Igualmente, los congresistas son definidos como Honorables e Ilustrados, en 13 ocasiones respectivamente: Honorables, por desempeñar el puesto de legisladores e Ilustrados porque saben qué actitud tomar, saben ser prudentes, tienen conocimientos o mucha experiencia. Entre otras expresiones se encuentra la siguiente referida a los legisladores: "Sois el pensamiento y la conciencia de la Patria".

Mediaciones Sociales, № 10, I semestre 2012, pp. 63-85. ISSN electrónico: 1989-0494. DOI: http://dx.doi.org/10.5209/rev_MESO.2012.n10.39682 


\section{Gráfico 1. Rasgos compartidos y no compartidos en las representaciones de CONGRESO y PRESIDENTE}

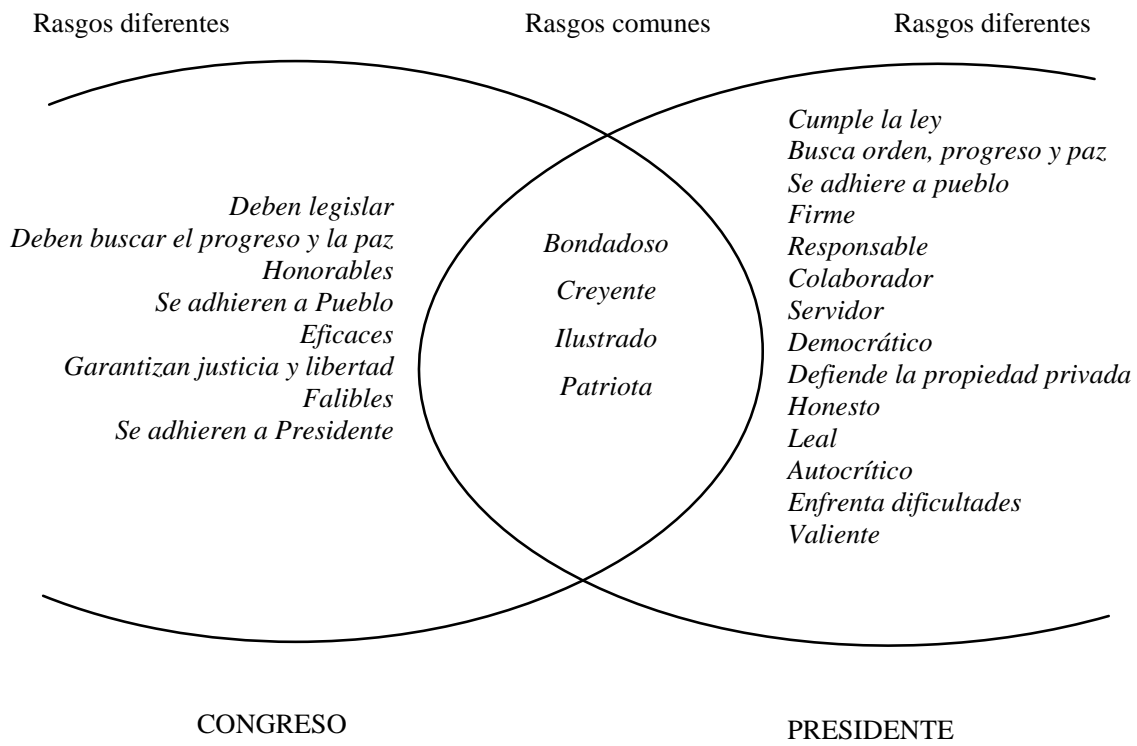

\subsubsection{La representación del PUEBLO}

José Tadeo Monagas adjudica 18 atributos distintos al referente PUEBLO, y de éstos el de mayor relevancia es el rasgo de Bondadoso (incluye expresiones de bondad, generosidad, magnanimidad, solidaridad y agradecimiento). Un PUEBLO bondadoso que sufre las calamidades de la guerra, analfabetismo, endemias y pobreza, pero que, sin embargo, es capaz de perdonar y ser solidario, de soportar sacrificios y enfrentar calamidades. Una representación que según los estudiosos seguía siendo común al pueblo venezolano para el siglo XX: "Durante muchos años, por lo menos durante casi toda la segunda mitad del siglo veinte, los venezolanos fuimos percibidos y nos vimos a nosotros mismos como una sociedad abierta, tolerante, integradora, inclusiva, hospitalaria" (Capriles, 2011: 136). 
También se dice del PUEBLO que es Democrático, Ilustrado y que Defiende y se adhiere al Gobierno (los tres rasgos, en 11 ocasiones). Según JTM, es democrático porque defiende y cree en el orden constitucional, porque goza de libertad y de los derechos y deberes de una patria libre, ama la libertad y la justicia, ama la democracia, ama a su país, ama los principios republicanos. Por otra parte, la cualidad de Ilustrado, paradójicamente se atribuye a un pueblo en su mayoría analfabeto, sujeto a desmanes del más poderoso, ubicado un $80 \%$ en áreas rurales sin recursos educativos y con ingresos apenas de subsistencia.

La atribución de rasgos negativos (Extraviado y Victima) sumados a las relaciones de dependencia que se le asignan a PUEBLO, puede ser explicada desde otro punto de vista: su debilidad lo hace depender de la conducción del mandatario y de los congresistas. Capriles afirma que a principios del siglo XX "se postuló la barbarie, la inmadurez o una incapacidad básica del pueblo venezolano como explicación y justificativo del gendarme necesario" (2011: 76), idea que pudo ser sembrada a partir de los años de inestabilidad, pobreza y atraso de la segunda mitad del siglo XIX. Con el PRESIDENTE, el PUEBLO comparte la creencia en Dios, la bondad, el conocimiento, el patriotismo y el amor por la democracia. 


\section{Gráfico 2. Rasgos compartidos y no compartidos en las representaciones de PUEBLO y PRESIDENTE}

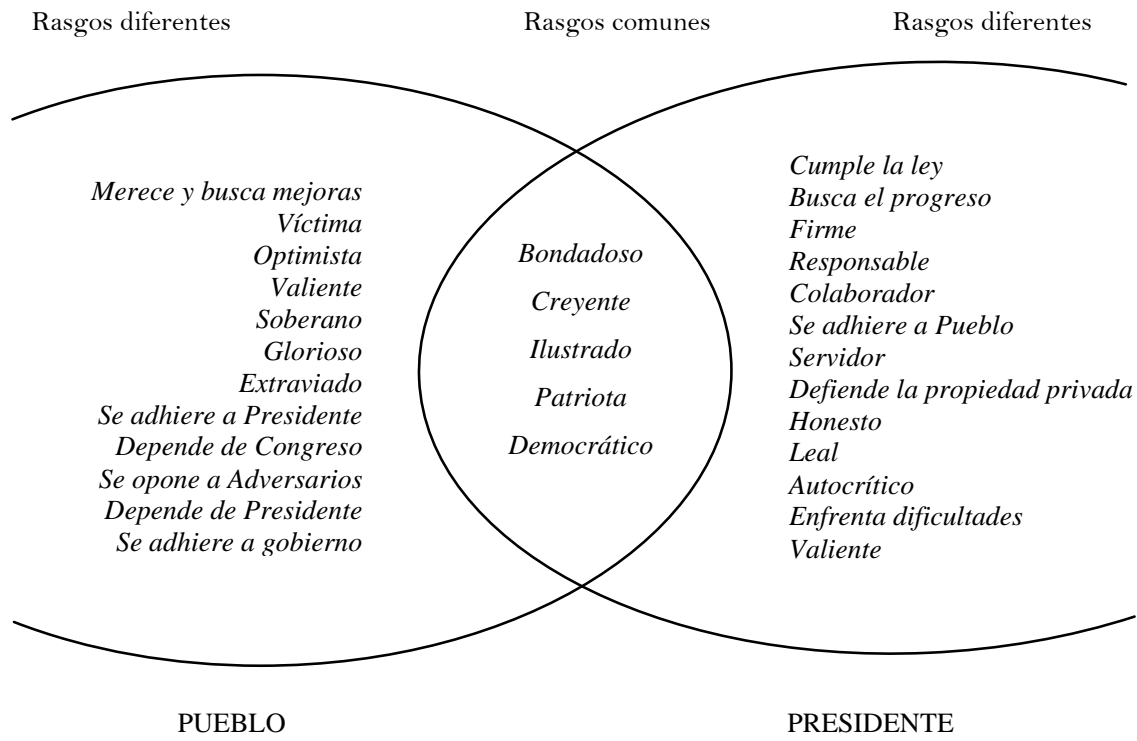

\subsubsection{La representación del PRESIDENTE}

La cualidad atribuida con mayor frecuencia al PRESIDENTE es la creencia en Dios. El propio José Tadeo Monagas, en el número de ocasiones señaladas (15), hace uso de frases donde coloca a Dios como testigo, pide la protección de la Providencia, manifiesta su fe en Dios, agradece al Creador, reconoce el poder celestial o se autodefine como creyente. Esta identificación en la fe es incluso más importante que la vinculación a la legalidad, al respeto y al cumplimiento de las leyes, rasgo que ocupa para el referente PRESIDENTE el segundo lugar junto con el ser patriota, pues cada una de ellas se vincula once veces a la figura del mandatario nacional. Por tanto, las representaciones creadas en torno a la figura presidencial estarán referidas más a los aspectos intangibles como la fe y el patriotismo y en menor medida a lo legal, a la honestidad o a la eficiencia. En un país que se levanta sobre los escombros de la guerra de la independencia y 
marcha paralelo a los levantamientos internos, su Presidente en primer lugar cree en Dios, luego ama a la Patria, la sirve y entrega su vida por ella y cumple y hace cumplir las leyes. Sin embargo, la responsabilidad de buscar orden, progreso y paz para el país, que la asigna en 20 ocasiones al CONGRESO, tan sólo se la adjudica a sí mismo en nueve ocasiones y señalando que el PRESIDENTE busca el progreso y la paz.

\subsubsection{La representación del PAÍS}

El atributo estabilidad se asigna en 16 ocasiones al referente PAÍS. Estabilidad es una categoría que se registra cuando se afirma que el país está en paz, que quiere la paz, que es pacífico, busca la paz o necesita la paz; se presenta como un país estable o que desea y busca la estabilidad. Las guerras internas, los enfrentamientos con caudillos o las sublevaciones de líderes militares están presentes durante casi los ocho años que gobierna Venezuela el general José Tadeo Monagas y a pesar de ello el ideal de paz y estabilidad sigue presente como representación de lo que el país es, o puede ser. Del PAÍS se dice, en segundo lugar, que busca el orden, el progreso y la paz, con lo cual se refuerza la imagen de estabilidad y trabajo por la paz que se dibuja de la nación en estos discursos. Es distinto el caso de la atribución de democrático, rasgo que solo se asigna al PAÍS en seis ocasiones aún cuando para mediados y finales del siglo XIX ya en el país se discutía la creación de un Estado liberal-democrático: "Pensamos que las raíces históricas del proceso democrático venezolano tienen un primer momento en la compleja discusión política ocurrida en Venezuela durante el siglo XIX" (Dávila, 1991, p. 10). La bondad y el apego al sistema democrático, así como su dependencia de otros actores son atributos comunes entre PUEBLO y PAÍS. 


\section{Gráfico 3. Rasgos compartidos y no compartidos en las representaciones de PUEBLO y PAÍS}

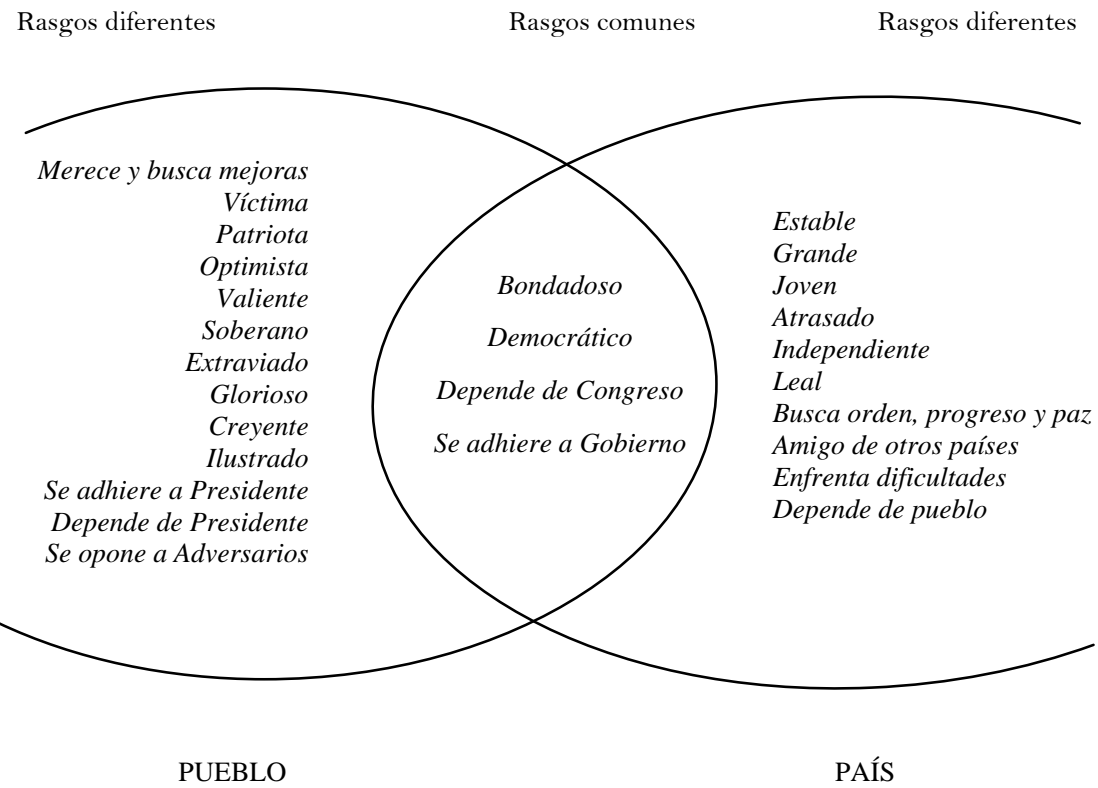

\subsubsection{La representación del GOBIERNO}

Con tal solo once rasgos José Tadeo Monagas construye una representación del GOBIERNO que está vinculada a la democracia, al respeto, a la firmeza y a la generosidad: a la democracia cuando anuncia que el GOBIERNO cumple y hace cumplir las leyes, se adhiere al pueblo, es democrático, respeta las instituciones, es dialogante; al respeto y la firmeza cuando señala que su GOBIERNO somete al enemigo, es justo, firme y enfrenta dificultades; $\mathrm{y}$, finalmente, a la generosidad cuando dice que es honesto, justo y generoso.

\subsubsection{La representación de los ADVERSARIOS}

ADVERSARIOS es una categoría de referente que reúne a enemigos alzados en armas, caudillos del pasado que siguen ocasionando problemas 
(José Antonio Páez, por ejemplo), escritores de oposición que escriben en los diarios e incluso el partido de oposición. De todos los atributos negativos que le adjudican el manipular la información y el oponerse al país, son los más abundantes, así es como ellos difaman al gobierno, siembran incertidumbre, calumnian, abusan de la libertad de prensa pero también: son criminales, egoistas, se oponen al Gobierno y al pueblo y son facciosos. Reúne este referente un grupo de atributos negativos que no se asigna a ningún otro.

\section{Algunas Conclusiones}

El análisis realizado con los rasgos atribuidos a los referentes seleccionados muestra que efectivamente los discursos pronunciados ante el Congreso de la República de Venezuela por José Tadeo Monagas entre los años 1847 y 1858 ofrecen unas representaciones de la realidad venezolana que guían y orientan la acción que desde el gobierno, la presidencia o la población se puede asumir ante los hechos ocurridos en su mandato. Unos hechos que, como se observa en la breve reseña histórica, están asociados a violencia, desequilibrios y desajustes y que, sin embargo, a través del discurso se neutralizan para ofrecer a la comunidad la seguridad y estabilidad del sistema vigente.

Retomemos ahora los tres objetivos que, a juicio del profesor Manuel Martín Serrano puede trazarse el uso funcional de los discursos mediadores: 1. Mejorar su eficiencia, 2. Disminuir o eliminar las disfunciones, y 3. Evitar o posponer el ajuste histórico necesario (Martín Serrano, 2004: 76). El modo en el cual los discursos del Presidente Tadeo intentan cubrir tales objetivos sería el siguiente:

1. Mejora la eficiencia del discurso presentando al adversario como enemigo único y ajeno a la sociedad, carente de argumentos válidos para su lucha y sin vinculación alguna con los referentes involucrados en el quehacer diario de un país. De manera evidente, la articulación "etnogrupo // exogrupo" se aplica en el discurso político. En este caso, el discurso del Presidente (de todos los venezolanos) ubica con nitidez la presencia de "los otros" como factor perturbador y agrupa como "nuestros" al resto de los actores.

Mediaciones Sociales, № 10, I semestre 2012, pp. 63-85. ISSN electrónico: 1989-0494. DOI: http://dx.doi.org/10.5209/rev_MESO.2012.n10.39682 
También intenta mejorar la eficiencia del discurso ignorando las responsabilidades u obligaciones del "presidente" y haciendo ver al "pueblo" que, a pesar de sus grandes cualidades como ser bondadoso, ha cometido equivocaciones, ha sido víctima y requiere del "congreso", del "gobierno" y del "presidente" para alcanzar el orden, el progreso y la paz. Esas propuestas sobre la realidad intentan orientar el comportamiento de la población ante los cambios objetivos de la sociedad venezolana descrita arriba.

2. Por otra parte, se apoya en principios universales como paz, democracia, bondad y conocimiento para disminuir las disfunciones y contradicciones que podría ocasionar tener que referirse a un país envuelto en guerra. El Presidente anuncia que pronto se conseguirá la paz o mantiene que el enemigo ha sido sometido. Destaca frecuentemente que el "pueblo" es bondadoso, democrático e ilustrado, cuando los venezolanos son mayoritariamente analfabetos, desconocedores de un régimen democrático estable y viven en una constante lucha por defender su vida del propio gobierno, de las clases económicas más poderosas, de los alzados en armas y hasta de las grandes epidemias que los aquejan.

3. Finalmente, no hay más ajuste que el consistente en suavizar y adecuar a los intereses de su gobierno la representación que da de cada uno de los referentes, y a través de ella, de la realidad venezolana. Cuando tiene que referirse al atraso, el desorden en la administración pública y la ineficacia del poder judicial, entre otros problemas disfuncionales, señala que se originan por la carencia de leyes para combatir tales males. Cuando se trata de la inestabilidad, culpa a líderes del pasado y a los inadaptados que quieren tomar el poder; lo que es tanto como decir: pídanle memoria y cuenta a los otros.

Con este avance de la investigación se observa como las articulaciones miembros // no miembros (en el plano espacial) y vigente // no vigente (en el plano temporal) señaladas por Martín Serrano (2008) alimentan los prejuicios y estereotipos que vinculan la seguridad individual y colectiva al etnocentrismo. Lo hace JTM cuando construye sus discursos sobre la base de quien está con ellos y quién está fuera, quien sueña como ellos y quien tiene sueños diferentes; excluye a aquellos que intenten ser diferentes y los tilda de peligrosos, criminales, igual si son escritores, revoltosos, sublevados en armas o mandatarios anteriores. Por ello destaca que los "otros" están influenciados por los de fuera, son ambiciosos e individualistas, pien- 
san solo en intereses personales, por ello se oponen al interés colectivo, al interés del pueblo, por tanto la seguridad de la colectividad y de la estructura de poder se encuentra en "los nuestros", quienes trabajan con el mediador [Jefe de Estado], reciben su ayuda, lo apoyan y, a la vez, necesitan del mediador para conseguir sus objetivos últimos, la felicidad, el progreso, la democracia.

Cabe concluir, por tanto, que el discurso político, al igual que la televisión busca el consenso social a partir de la exclusión de todo lo extraño al grupo (al gobernante en este caso), sean valores, acontecimientos, propuestas, acciones. En general, los discursos políticos son creados para orientar el funcionamiento de la sociedad y de las personas de acuerdo con algún propósito, designio o concepción del mundo, que se legitiman en el propio discurso. Por ello organizan la información con el fin de pautar conocimientos y comportamientos que lleven a la sociedad y a las personas a ese fin preconcebido.

\section{BIBLIOGRAFÍA}

Alarico Gómez, C. (2006): José Tadeo Monagas. Caracas: Biblioteca Biográfica Venezolana / Diario El Nacional / Banco del Caribe.

Arráiz LuCCA, R. (2008): Venezuela: 1830 a nuestros días. Caracas: Alfa.

BARDin, L. (2002): Análisis de contenido. Madrid: Akal.

BERnETE, F. (1990): Los estereotipos de la comunidad iberoamericana: La representación de los personajes en los libros de texto de historia. Madrid: Universidad Complutense de Madrid. Tesis Doctoral.

BERNETE, F. (1992): "El estudio de los estereotipos a través del análisis de relatos”, Revista Española de Investigaciones Sociológicas, nº 57, pp. 123-135.

CAPRILES, A. (2011): Las fantasías de Juan Bimba. Mitos que nos dominan, estereotipos que nos confunden. Caracas: Taurus.

DÁvila, L. R. (1991): La democracia venezolana orígenes ideológicos y sociales. Mérida, Venezuela: Universidad de Los Andes, Consejo de Desarrollo Científico, Humanístico y Tecnológico, Consejo de Publicaciones. 
Martín Serrano, M. (1978): “Técnica de análisis de la mediación”, en MarTín Serrano, M. et al.: Métodos de análisis de contenido. Madrid: Facultad de CC. de la Información-Universidad Complutense de Madrid.

Martín Serrano, M. (2004): La producción social de comunicación. Madrid: Alianza.

Mangano Molero, F. (2010): “Alianzas y vínculos de solidaridad. Páez y la élite maracaibera”, CONHISREMI. Revista Universitaria Arbitrada de Investigación y Diálogo Académico, vol. 6, $\mathrm{n}^{\circ}$ 2, pp. 56-68. Disponible en http://conhisremi.iuttol.edu.ve/pdf/ARTIO00096.pdf. Consultado el 12 de febrero de 2012.

SABINO, C. (1995): "El sistema político venezolano: estabilidad, crisis e incertidumbre”, Contribuciones, año XII, $\mathrm{n}^{\circ}$ 1, pp. 149-167. Disponible en http://paginas.ufm.edu/sabino/word/Articulos_capitulos_de_libros_monografias _ensayos/sistema_politico_venezolano.pdf. Consultado el 12 de diciembre de 2011.

Rodríguez Mirabal, A. (2002): “Monagas, José Tadeo”, en Diccionario de Historia de Venezuela. Caracas: Fundación Polar, pp. 223-226. 


\section{PARA CITAR ESTE TRABAJO EN BIBLIOGRAFÍAS:}

VALECILLOS VÁZQUEZ, Carmen y BERNETE, Francisco (2012): "Representaciones de la sociedad venezolana en los discursos del Presidente de Venezuela José Tadeo Monagas”, Mediaciones Sociales. Revista de Ciencias Sociales y de la Comunicación, nº 10, pp. 63-85. DOI: http://dx.doi.org/10.5209/rev_MESO.2012.n10.39682

\section{${ }^{(*)}$ Los autores}

Carmen Valecillos Vázquez es profesora del área de Formación Periodística en la Universidad de Los Andes-Táchira Venezuela. Actualmente realiza el doctorado de Comunicación Social en la Universidad Complutense de Madrid. Ha recibido varios reconocimientos, entre ellos, PEI 2001, PPI 2008, y mejor docente en Premio Regional de Periodismo en el 2005. Dentro de sus publicaciones más recientes se encuentran: Valecillos (2008): Descripción del tratamiento informativo dado al hecho económico fronterizo en el diario La Nación de San Cristóbal; Valecillos y Henríquez (2008): El periódico digital laboratorio, alianza entre la formación teórica y la práctica profesional del periodista (2008); Henríquez y Valecillos (2008): Estudio diagnóstico sobre hábitos y consumo de periódicos digitales para la construcción de un medio digital estudiantil.

Francisco Bernete es doctor en Ciencias de la Información. Profesor Titular de Teoría y Sociología de la Comunicación en la Universidad Complutense de Madrid. En posgrado, imparte docencia relacionada con el análisis de contenido y la comunicación pública en la sociedad multiétnica. Ha participado en varias investigaciones y publicaciones donde se relaciona la comunicación con la juventud, los estereotipos o las identidades. Ha sido director de la Sección Departamental de Sociología IV en la Facultad de Ciencias de la Información y actualmente es coordinador del Máster Oficial en Comunicación Social de la UCM.

RECIBIDO: 20 de febrero de 2012.

ACEPTADO: 24 de junio de 2012.

Mediaciones Sociales, № 10, I semestre 2012, pp. 63-85. ISSN electrónico: 1989-0494. DOI: http://dx.doi.org/10.5209/rev_MESO.2012.n10.39682 
